\title{
Quality Control Based on Isotopic Distributions for High-Throughput MALDI-TOF and MALDI-FTICR Serum Peptide Profiling
}

\author{
Simone Nicolardi, ${ }^{\text {a }}$ Magnus Palmblad, ${ }^{\text {a }}$ Hans Dalebout, ${ }^{\text {a }}$ \\ Marco Bladergroen, ${ }^{a}$ Rob A. E. M. Tollenaar, ${ }^{\text {b }}$ André M. Deelder, ${ }^{\text {a }}$ and \\ Yuri E. M. van der Burgt ${ }^{a}$ \\ ${ }^{a}$ Department of Parasitology, Biomolecular Mass Spectrometry Unit, Leiden University Medical Center \\ (LUMC), Leiden, The Netherlands \\ ${ }^{\mathfrak{b}}$ Department of Surgery, Leiden University Medical Center (LUMC), Leiden, The Netherlands
}

In this study, we have implemented a new quality control (QC) parameter for peptide profiling based on isotopic distributions. This QC parameter is an objective measure and facilitates automatic sorting of large numbers of peptide spectra. Peptides in human serum samples were enriched using reversed-phase $\mathrm{C}_{18}$-functionalized magnetic beads using a high-throughput robotic platform. High-resolution MALDI-TOF and ultrahigh resolution MALDI-FTICR mass spectra were obtained and a workflow was developed for automated analysis and evaluation of these profiles. To this end, the isotopic distributions of multiple peptides were quantified from both MALDI-TOF and MALDI-FTICR spectra. Odd peptide isotope distributions in TOF spectra could be rationalized from ultrahigh resolution FTICR spectra that showed overlap of different peptides. The comparison of isotope patterns with estimated polyaveragine distributions was used to calculate a QC value for each single mass spectrum. Sorting these QC values enabled the best MALDI spectrum to be selected from replicate spots. Moreover, using this approach spectra containing high intensities of polymers or other contaminants and lacking peptides of interest can be efficiently removed from a clinical dataset. In general, this method simplifies the exclusion of low quality spectra from further statistical analysis. (J Am Soc Mass Spectrom 2010, 21, 1515-1525) (c) 2010 American Society for Mass Spectrometry

$I^{\prime}$ n the past decade, discovery studies in clinical proteomics have evolved synergistically with the technological progress made in mass spectrometry (MS). The growth in the field of MS-based proteomics $[1,2]$ has been driven by the introduction of new identification techniques $[3,4]$ and improvements in the resolution, mass accuracy, robustness, and dynamic range of modern mass analyzers [5-8]. From the early days, serum peptide- and protein profiling has played a major role in clinical studies for biomarker discovery, aiming at identifying differences in peptide- and protein levels in serum of healthy and diseased individuals [9-13]. Peptide and protein patterns can change as a result of disease and are thus helpful in both early detection and monitoring the development of the disease. However, serum is a very complex mixture of biomolecules and, therefore, requires appropriate sample workup. In addition to the complexity, the peptide and protein profiles of serum are usually dominated by highly abundant species (the tip of the iceberg) [14]. Different strategies have been followed to reduce the

Address reprint requests to Dr. Y. E. M. van der Burgt, Department of Parasitology, Biomolecular Mass Spectrometry Unit, Leiden University Medical Center, Albinusdreef 2, P.O. Box 9600, Zone L4-Q 2300 RC Leiden, The Netherlands. E-mail: Y.E.M.van_der_Burgt@lumc.nl biological complexity of human serum samples and multiple methods and technologies have been developed for the purpose of sample workup [15-19]. The combination of protein and peptide extraction using functionalized magnetic beads with matrix-assisted laser desorption/ionization (MALDI) MS has been widely used for profiling studies [20-22]. One of the advantages of this approach is that automation of both solid-phase extraction (SPE) and MS allows highthroughput screening. Also, for each serum sample, fresh disposable magnetic beads are used, thus avoiding carry-over that may occur when other techniques such as liquid chromatography (LC) are used. Moreover, magnetic beads with a different functionality allow protein- and peptide enrichment based on different chemical-physical interactions, thus broadening the range of components covered.

Several studies have focused in detail on sources of variance in profiling experiments and the necessity of standardization in sample collection and preparation has been demonstrated [23-25]. Robotic liquid handling has the advantage of better reproducibility of the SPE and a greatly increased throughput. Robotic platforms enable a fully automated use of magnetic beads and ensure the control of each step in the extraction protocol 
[26-28]. In addition, the high speed of data acquisition of MALDI MS allows the analysis of thousands of samples in a realistic time period. The application of statistical analysis for the evaluation of the high number of data generated using this strategy, finally enables a more confident identification of possible biomarkers.

In profiling studies, the most commonly used mass analysis in combination with MALDI is time-of-flight mass spectrometry (TOF-MS) [29-31]. Following the current developments in high-resolution mass spectrometry, MALDI coupled with Fourier transform ion cyclotron resonance (FTICR-MS) has been applied for the same purpose $[32,33]$. The high-resolution and low or even sub-ppm mass measurement errors result in more confident peptide and protein assignments. In this study, we compared peptide profiles using the two MS-platforms indicated above, i.e., MALDI-TOF and MALDI-FTICR. With respect to large-scale mass spectrometric proteomic or peptidomic profiling experiments, the quality control (QC) of both the workflow and the data yielded is an arduous task. Variations can occur pre-analytically (blood collection, clotting time, storage conditions), in the analytical workflow, and in the data acquisition (MS experiments). The human eye is extremely good at differentiating multiple pictures, e.g., peptide profiles. However, the visual inspection of all acquired spectra would be time-consuming and subjective. For this reason, various QC procedures and tools have been developed that allow (automated) evaluation of peptide- and protein MS profiles [34-38]. Generally, the signal to noise ratio and/or the threshold of the noise level are taken into account, whereas in MALDI MS protein and peptide profiling the reproducibility of the signal intensity is rigorously evaluated [39, 40]. The use of a highly robust protocol including replicate measurements for each sample allows further standardization. The evaluation of noise levels of different datasets (e.g., different MALDI plates) is more cumbersome and prone to bias. In this study, we describe a novel QC approach for the automatic selection of mass spectra, based on peptide isotopic distributions. To this end, the previously used model amino acid averagine was used to carry out detailed comparisons [41]. A recently developed integration method was used to obtain intensity values for each isotope signal within a given distribution. We will show that this QC parameter can clearly discriminate good and poor peptide spectra.

\section{Materials and Methods}

\section{Blood Collection and Serum Handling}

Blood samples were collected from patients and healthy volunteers by antecubital venipuncture while the person was seated. Informed consent was obtained from all patients, and the Leiden University Medical Center (LUMC) Medical Ethical Committee approved the studies. All clinical studies were carried out under supervi- sion of the Department of Surgery at the LUMC. It is outside the scope of this paper to give a detailed description of the various patient cohorts. Subsets of the data will be reported elsewhere.

Within $1 \mathrm{~h}$ after collection of blood in a $10 \mathrm{~mL} \mathrm{BD}$ vacutainer tube (containing a clot activator and a gel for serum separation) the sample was centrifuged for 10 $\min$ at $1500 \times g$. The serum was then transferred to a 1 $\mathrm{mL}$ polypropylene cryovial, frozen, and stored at $-80^{\circ} \mathrm{C}$. Each cryovial was thawed only once and divided over eight $50 \mu \mathrm{L}$ Matrix 2D barcoded storage tubes (Thermo Fisher Scientific Inc., Hudson, NH, USA) using an 8-channel Hamilton pipetting robot. These tubes were then distributed over eight different 96sample matrix latch racks, thus yielding eight identical Matrix racks containing 96 different serum samples. These racks were frozen again at $-80^{\circ} \mathrm{C}$ and a rack was thawed only once for the automated peptide capture procedure. The sample thawing time, i.e., the time between taking a rack out of the freezer and the starting time of the peptide isolation procedure (next section), was between 30 and $60 \mathrm{~min}$ for all serum samples. This high degree of standardization in sample handling and sample thawing-time in combination with the use of the 96-channel pipetting robot ensured identical (robust) and simultaneous treatment of all serum samples in the peptide isolation procedure.

\section{High-Throughput Peptide Isolation with RPC18 Dynabeads and MALDI Spotting}

The isolation of peptides from human serum was performed using reversed-phase $\mathrm{C}_{18}$-functionalized magnetic beads [11, 26, 27]. For each sample, a fresh suspension of paramagnetic monodisperse beads was used and all 96 serum samples in one Matrix rack were incubated with the same lot-number of beads. In this study $10 \mu \mathrm{L}$ of commercially available RPC18 Dynabeads (Invitrogen, Carlsbad, CA, USA) was used for the analysis of $5 \mu \mathrm{L}$ of undiluted human serum. The activation, wash, and desorption steps of the RPC18 beads were based on the manufacturer's protocol; however, the protocol was adjusted to enable an optimal implementation on a 96-channel Hamilton STARplus pipetting robot (Hamilton, Bonaduz, Switzerland). In short, a plate containing 96 magnets was designed and built in-house to accommodate a 96-well PCR microtiter plate (MTP) and to allow for optimal settlement of the magnetic beads at the bottom of each well. Furthermore, it was found that additional activation steps were needed, since automated pipetting does not allow the removal of all liquid from the pulled-down magnetic beads. Thus, for optimal removal of preservatives during the activation step the RPC18 beads were washed three times with $50 \mu \mathrm{L}$ of water. For similar reasons, after binding of the peptides to the RPC18 beads three wash steps with $50 \mu \mathrm{L}$ of a $0.1 \%$ trifluoroacetic acid solution were carried out. Finally, the eluates obtained 
in the peptide desorption step (performed with a 50\% acetonitrile solution) were transferred into a 96-well plate and mixed with stabilization buffer. A portion of these eluates $(2 \mu \mathrm{L})$ was used for MALDI-spotting while the remainder was frozen and stored at $-80^{\circ} \mathrm{C}$ for future analysis.

The 96-channel Hamilton STARplus pipetting robot was used for mixing of sample eluates with MALDImatrix, followed by spotting on a MALDI target plate. To this end, $2 \mu \mathrm{L}$ of the stabilized eluate was transferred into a fresh 384-well MTP and mixed with $10 \mu \mathrm{L}$ of $\alpha$-cyano-4-hydroxycinnamic acid $(0.3 \mathrm{~g} / \mathrm{L}$ in ethanol: acetone 2:1). Four MALDI spots of each eluate were obtained after quadruplicate spotting on a MALDI AnchorChip (600 $\mu \mathrm{m}$; Bruker Daltonics, Bremen, Germany) using $1 \mu \mathrm{L}$ of the eluate/matrix mixture for each spot.

\section{MALDI-TOF and -TOF/TOF Mass Spectrometry}

After MALDI spotting, the target plate was immediately stored in a storage chamber (RT, 5\% oxygen, 95\% nitrogen), until transfer into the MALDI-TOF instrument by a CRS F3 robotic arm (Thermo Fisher Scientific Inc., Marietta, OH, USA), as a fully automated plate loader [42]. In this way, all MALDI-TOF measurements were carried out within $12 \mathrm{~h}$ after spotting on the 96-channel robot. All MALDI-TOF experiments were performed on an UltraFlex III (Bruker Daltonics) operating in positive reflectron mode in the $m / z$ range of 600-4000. The spectra were acquired using FlexControl software ver. 3.0 (Bruker Daltonics) with identical data acquisition parameters. A SmartBeam $200 \mathrm{~Hz}$ solidstate laser, set at a frequency of $100 \mathrm{~Hz}$, was used for ionization. A profile, or summed spectrum, was obtained for each MALDI-spot by adding 20 spectra of 60 laser shots each at different rasters. FlexControl software decided on-the-fly whether or not a scan was used for the summed spectrum. To this end, a resolution higher than 2000 was required. Peaks were detected using the SNAP centroid peak detection algorithm with signal-to-noise threshold of 1 and a "TopHat" baseline subtraction. All mass scans not fitting these criteria were excluded. The measurement of a MALDI spot was finished when 1200 laser shots had been summed in one profile. The MALDI-TOF spectra were measured from $\mathrm{m} / \mathrm{z} 600$ to 4000 and externally calibrated using a commercially available peptide mix (Bruker Daltonics). FlexAnalysis Software 3.0 (Bruker Daltonics) was used for visualization and initial data processing.

For identification, the LIFT-TOF/TOF spectra were recorded on the same Bruker Ultraflex III TOF/TOF mass spectrometer. No additional collision gas was applied. Precursor ions were accelerated to $8 \mathrm{kV}$ and selected in a timed ion gate. The fragments were further accelerated by $19 \mathrm{kV}$ in the LIFT cell, and their masses were analyzed after the ion reflector passage.

\section{Peak Alignment in MALDI-TOF Serum Peptide Profiles}

The workflow for processing peptide profiles is depicted in Figure 1. After external calibration using a commercially available peptide mix, the maximum observed mass shift in the 384 spectra varied from $\sim 0.1 \mathrm{Da}$ at $m / z 1500$ to $0.2 \mathrm{Da}$ at $m / z 4000$. Before carrying out the alignment a baseline subtraction of all spectra was performed. To allow the alignment of all 384 spectra from one MALDI target plate at least three peptides at different $\mathrm{m} / \mathrm{z}$ values were needed for internal calibration. To compensate for the possible absence of one or two peptides in a spectrum, the following five peptides were selected based on a manual inspection of a few spectra, namely $m / z$ 1465.8, 1778.1, 1865.2, 2602.5, and 2931.5, with a tolerance window of $100 \mathrm{ppm}$ for the $\mathrm{m} / \mathrm{z}$ 1465.8 peak increasing up to $300 \mathrm{ppm}$ for the highest $\mathrm{m} / \mathrm{z}$ value (FlexAnalysis 3.0).

\section{Determination of Peak Intensities Using Xtractor}

All MALDI-TOF and the MALDI-FTICR spectra were exported as DAT (.dat) and XY (.xy) files, respectively. In both formats (ASCII), all $\mathrm{m} / \mathrm{z}$ values with corresponding intensities (i.e., data points) were reported. The simple Xtractor tool was used to determine the intensity of each user-defined peak [43]. To this end, a certain $\mathrm{m} / \mathrm{z}$ window was defined for each peak included in a so-called reference file, thus allowing Xtractor to sum all the intensities of the data points within the defined bin. The bin size for all peaks in the MALDI-TOF spectra was fixed at $\pm 0.49 \mathrm{Da}$, the bin size for the peaks

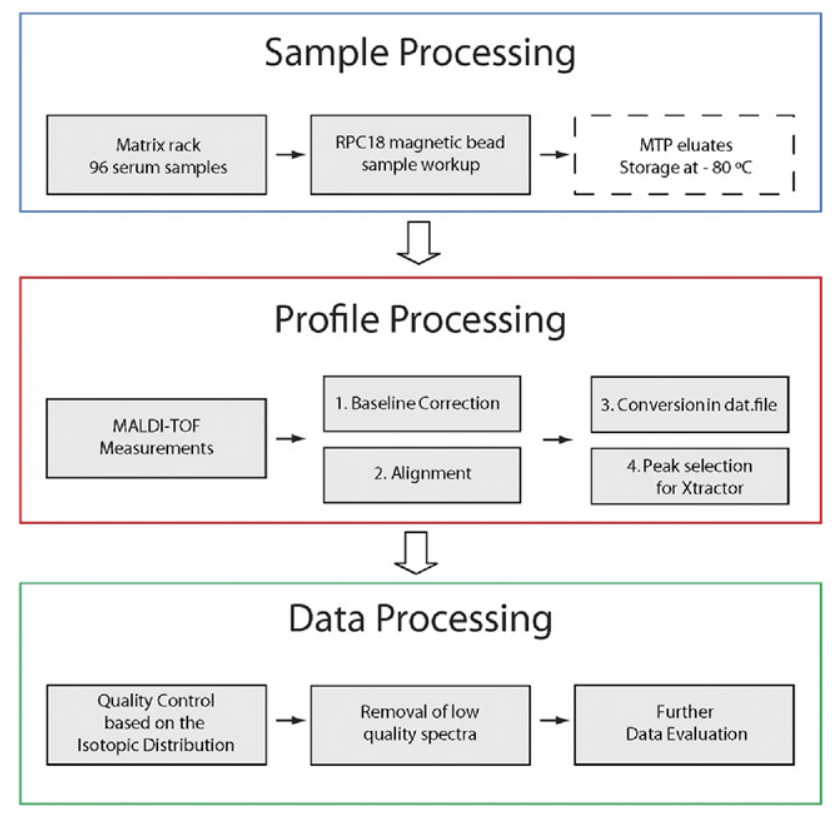

Figure 1. Overview of three sequential processing methods for the generation and evaluation of human serum peptide profiles. The novel QC method reported here is carried out in the third step. 
in the MALDI-FTICR spectra varied from $\pm 0.02 \mathrm{Da}$ at $\mathrm{m} / \mathrm{z} 1465.8$ to $\pm 0.08 \mathrm{Da}$ at $\mathrm{m} / \mathrm{z} 2931.5$. In this way, single intensity values for each isotopic peak within any isotope distribution were obtained. Importantly, Xtractor generates uniform data (peak) arrays regardless of spectra content (Xtractor is an open source tool and can be found at www.ms-utils.org/Xtractor).

\section{Calculation of Peak Intensities in Peptide Isotope Distributions}

The observed peptide isotopic distributions were compared with those obtained from the polyaveragine model as explained in Results and Discussion section. The theoretical isotopic distribution of a polyaveragine peptide was calculated using chemcalc (www.chemcalc. org). The elemental composition used for the calculation of peak intensities of each individual isotope reflected a singly protonated polyaveragine peptide molecule with integer numbers for all indices of the atoms $\mathrm{C}, \mathrm{H}, \mathrm{N}, \mathrm{O}$, and $\mathrm{S}$. In a similar way, the theoretical isotopic distribution of the peptide at $\mathrm{m} / \mathrm{z} 1465.8$ was calculated after identification of this peptide using MALDI-TOF/TOF. Note that each individual isotope within a distribution was considered as one single peak, as described earlier by Rockwood et al. [44] Thus, by using the jcamp file from chemcalc, all calculated intensities within the isotopic fine structure of the second and third peak were summed to two individual values only.

\section{MALDI-FTICR Mass Spectrometry}

All MALDI-FTICR experiments were carried out on a Bruker Daltonics apex-ultra 9.4 tesla FTICR mass spectrometer. This instrument was equipped an Apollo II dual ion source, two ion funnels, a selection quadrupole in the front-end, and an Infinity ICR cell. For the purpose of comparing FTICR-measurements with results obtained from the TOF, manual MALDI-spotting was carried out from a frozen 96-well eluate plate. After thawing the RPC18 eluates at room-temperature, $2 \mu \mathrm{L}$ of each eluate was transferred into a 384-well plate and mixed with $10 \mu \mathrm{L}$ of $\alpha$-cyano-4-hydroxycinnamic acid $(0.3 \mathrm{~g} / \mathrm{L}$ in ethanol:acetone 2:1). Finally, $1 \mu \mathrm{L}$ of this mixture was manually spotted on a MALDI AnchorChip $(600 \mu \mathrm{m})$ in duplicate using a standard Gilson P2 pipette. In this way, 192 spots of 96 different samples were obtained. These spots were measured on the FTICR using a SmartBeam $200 \mathrm{~Hz}$ solid-state laser, operated a frequency of $100 \mathrm{~Hz}$. The irradiation spot size was $\sim 200 \mu \mathrm{m}$. The ions generated from 50 laser shots were accumulated in the hexapole and then transferred through the quadrupole to the collision cell. This process was repeated eight times per example on different raster spots. The 450 laser shots accumulated in the collision cell were then transferred to the ICR cell for mass analysis. The quadrupole was set to an $m / z$ of
400 and RF amplitude of $3000 \mathrm{~V}_{p-p}$. Each spectrum was generated by accumulation of eight scans with $512 \mathrm{~K}$ data points. All data were acquired using the Bruker ApexControl software and evaluated using Bruker DataAnalysis software.

\section{Results and Discussion}

\section{Peptide Isotope Distributions in MALDI-TOF Spectra}

The workflow for sample, profile, and data processing is depicted in Figure 1. One of the aims of this study was to demonstrate the use of peptide isotopic distributions of peptides for quality control parameter. For illustration, the isotopic patterns of the five ubiquitous peptides used for alignment are shown in Figure 2. While the selection of these five peptides was an arbitrary process, no software is available that yields a better result without the need of user-defined input parameters. One of these peptides was identified, namely $m / z 1465.8$, as a fragment of fibrinogen $\alpha$-chain (P02671, peptide DSGEGDFLAEGGGVR). Previous reports corroborate this identification [45]. Using the elemental composition of this peptide the theoretical isotopic distribution was calculated (see the Materials and Methods section). It should be emphasized, however, that identification of the peptides is not necessary for the evaluation of isotopic distributions, but clearly becomes important at the stage a biomarker candidate has been detected. Different strategies, followed by adjustments, have been proposed for the estimation of isotopic distributions of unidentified peptides [36, 46, 47]. In this study, we used the "averagine" model, proposed by Senko et al. to construct peptides from a hypothetical average amino acid [41]. This is sufficient for the estimation of isotopic distribution of unknown peptides since we are not searching for the best fit, but only need to discriminate between peptide and nonpeptide (e.g., polymer, matrix cluster) distributions. The estimated values of the five peptides are depicted in Figure 2. These are obtained by first transferring a peptide $m / z$ value into a neutral mass (i.e., $[\mathrm{m} / \mathrm{z}$ valueproton-water]), then dividing this number by the exact mass of averagine (i.e., $111.0543 \mathrm{Da}$ ), and multiplying the result with the indices of the molecular formula of averagine (i.e., $\mathrm{C}_{4.9384} \mathrm{H}_{7.7583} \mathrm{~N}_{1.3577} \mathrm{O}_{1.4773} \mathrm{~S}_{0.0417}$ ). Finally, one "water-molecule" and a proton are added to create the corresponding "polyaveragine" molecular formula and $m / z$ value. Due to rounding of the indices, the $m / z$ values of the observed peptide and the corresponding polyaveragine are not identical. It is clear from Figure 2 that the isotopic patterns of the five peptides used for alignment were in good agreement with the polyaveragine distributions. A quantitative evaluation of these isotope distributions will be given in the next section. Differences with estimated distributions may be indicative of the presence of peptides that contain post- 
Number of Averagine Residues

(a) 1000

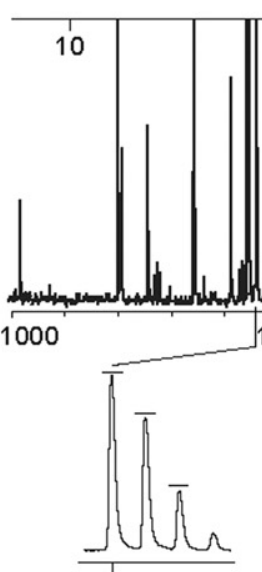

(b)

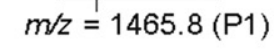

1500
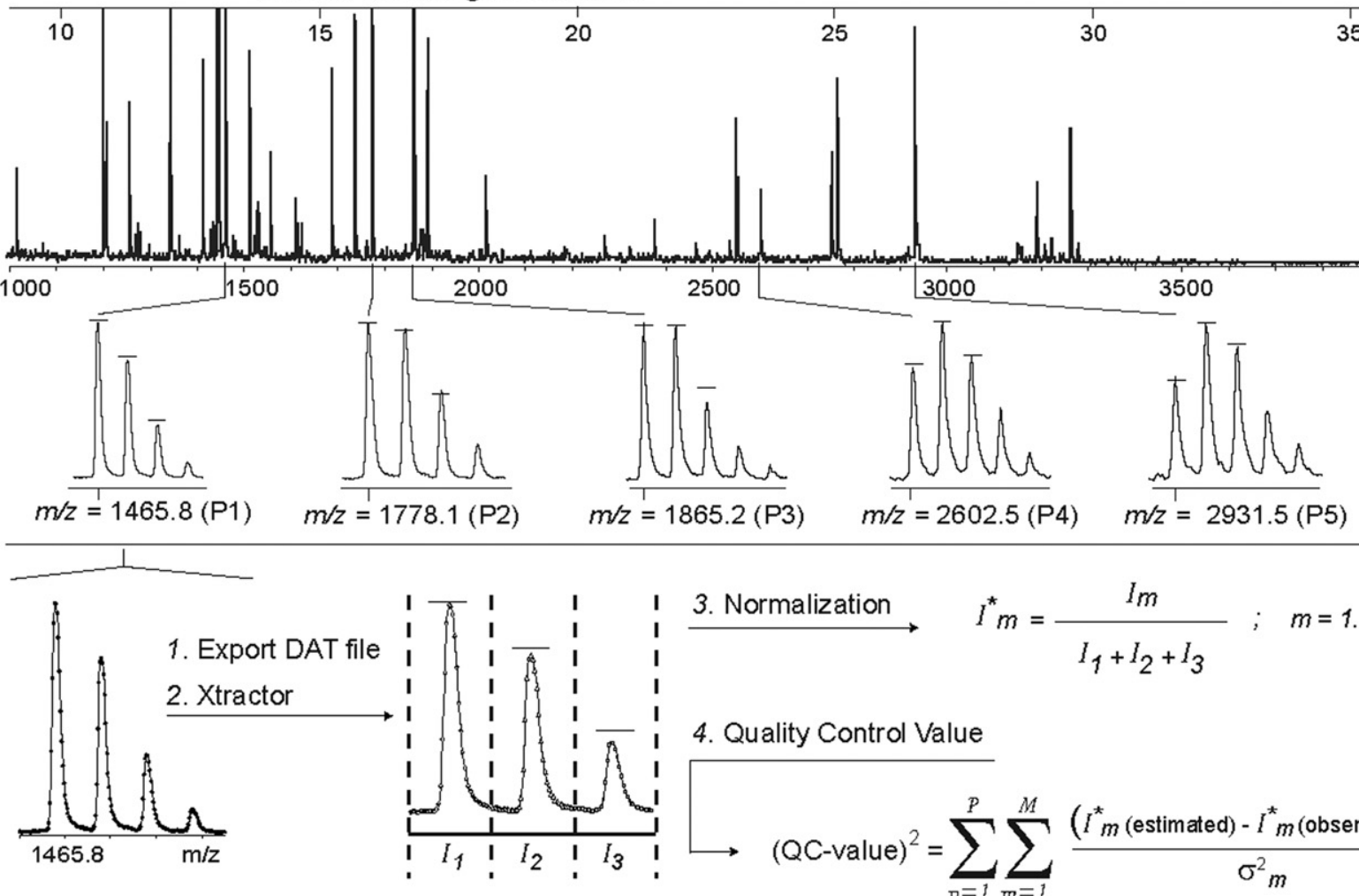

(c)

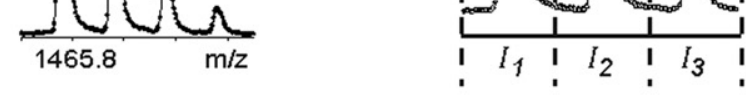

35

Figure 2. (a) MALDI-TOF peptide profile obtained from human serum after sample workup using RPC18-functionalized magnetic beads. (b) Observed isotopic distributions of five peptides chosen for alignment of all serum peptide profiles. The horizontal lines at the top of the peaks represent the calculated values of the corresponding polyaveragine peptides. (c) Profile processing results in a QC value for each individual mass spectrum. First, the intensity of each isotope peak is determined using Xtractor. Then, after normalization, the observed intensities are compared with those estimated from the polyaveragine peptide. For a more accurate determination of the deviation, the random noise variance is taken into account. Finally, the sum of thus obtained deviations for all considered peptides represents the QC value of the MALDI-TOF peptide profile in (a).

translational modifications (PTMs) or metal cations, or may point at overlapping peptides. Two examples of the latter cause of aberrant isotopic distributions are revealed in Figure 3a. Here, the isotope distributions of two peptides present in some of the MALDI-TOF profiles did not match those of the corresponding polyaveragine peptides. Subsequent high-resolution FTICR mass analysis of the same $\mathrm{m} / \mathrm{z}$ values clearly showed overlap of two different peptides. Further analysis showed that the weighted sum of two estimated polyaveragine distributions (with a mass difference of one $\mathrm{Da}$ ) matched the distributions observed in the TOF spectra. Differences with estimated distributions can be also indicative of sample contamination, such as polymers [48]. An example of MALDI-FTICR spectrum that contains polymer Tinuvin622 is shown in Figure 3b. The comparison of the isotopic distribution of the polymer at $\mathrm{m} / \mathrm{z}=1732$ with that estimated from a polyaveragine peptide with an $\mathrm{m} / \mathrm{z}$ of 1787 clearly shows that the species at $m / z=1732$ is not a peptide.

\section{Quality Control Based on Peptide Isotope Distributions}

The intensities of the observed peaks in each peptide isotope distribution were determined using Xtractor (see the Materials and Methods section). As an example, for the peptide at $\mathrm{m} / \mathrm{z} 1865.22$ the intensity of the monoisotopic was determined as the sum of all measured values at $m / z 1865.22 \pm 0.49$, the intensity of the second isotope was determined as the sum of all measured values at $m / z 1866.22 \pm 0.49$, and so forth (Figure 2). The window of $0.49 \mathrm{Da}$ was chosen to ensure total quantification of each isotope. This window of 0.49 Da does not reflect the actual peak width, i.e., the resolution of the peptides in the MALDI-TOF spectra varied from about 7000 at $m / z 1465.8$ and about 10,000 at $\mathrm{m} / \mathrm{z} 2$ 2931.5. It should be noted that the density of data points decreases with increasing $\mathrm{m} / \mathrm{z}$ values in a TOF spectrum. As a result, the number of data points within each bin differs between different peptides. However, within one peptide isotopic pattern, the number of data 

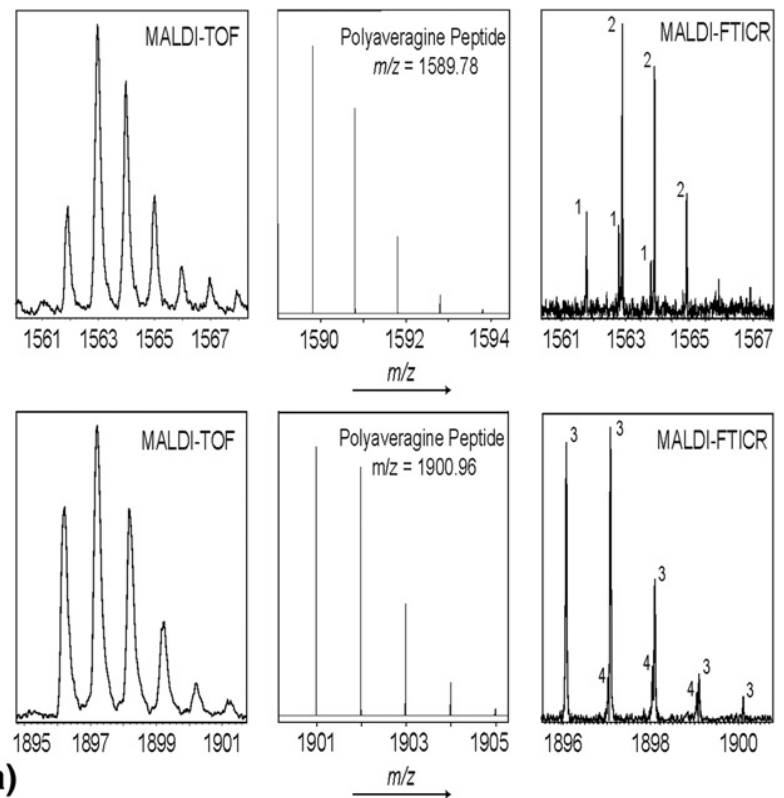

(a)

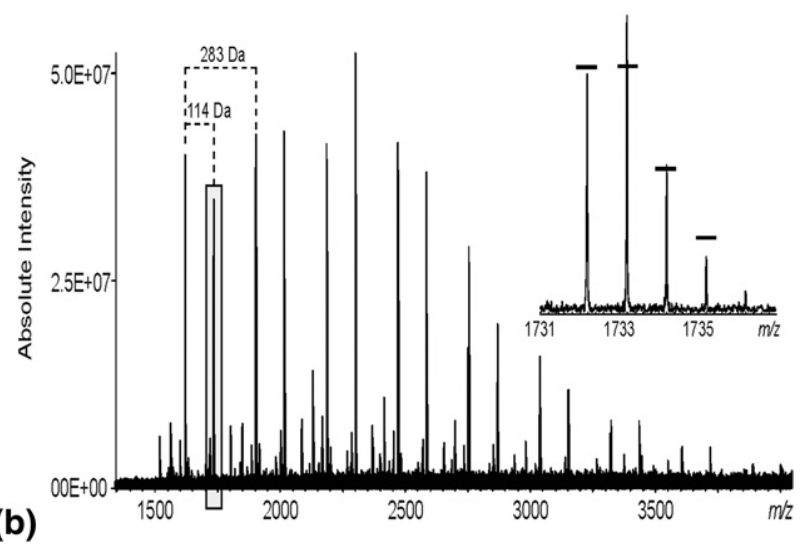

Figure 3. (a) Examples of deviations from the polyaveragine model due to overlap of different peptides. Left panel: enlarged part of a MALDI-TOF human serum peptide profile showing odd isotopic distributions. Middle panel: calculated isotope distributions of the corresponding polyaveragine peptides. Right panel: enlarged part of the MALDI-FTICR mass spectrum of the same spot reveals the presence of overlapping peptides 1 and 2 that cause the odd isotopic distribution in the MALDI-TOF spectra, and overlapping peptides 3 and 4. (b) Example of deviation from the polyaveragine model due to different peak identity. A MALDI-FTICR spectrum dominated by polymers of tinuvin-622 (poly-(N- $\beta$-hydroxyethyl-2,2,6,6-tetramethyl-4-hydroxy-piperidinyl succinate). The enlarged part shows the isotopic distribution of the polymer $\mathrm{m} / \mathrm{z} 1732$ and the isotopic distribution calculated from a polyaveragine peptide with $\mathrm{m} / \mathrm{z} 1787$ (horizontal bars).

points is the same for each single isotopic peak, at least for the five peptides considered here.

Three intensity values were obtained for all five peptides previously discussed in the spectral alignment part using the Xtractor tool. All isotope intensities were then normalized using the sum of the first three intensities within the distribution. These intensities were compared with the corresponding abundances in a polyaveragine isotopic distribution, as previously de- scribed by Palmblad et al. [49]. Here, the total signal variance is taken into account in the parameter $\sigma_{m}^{2}$. In the aforementioned study, carried out using electrospray ionization (ESI) FTICR-MS, $\sigma_{m}^{2}$ contained two terms, namely, experimental signal variance, which was approximately proportional to signal strength, and noise variance, which was constant. In MALDI spectra; however, every single laser shot yields a different amount of ions. As a result, the noise levels are not constant. In general, in the MALDI-TOF experiments performed in this study the noise variance decreased at increasing $\mathrm{m} / \mathrm{z}$ values, whereas the opposite was true for the MALDI-FTICR spectra. For the purpose of biomarker discovery and subsequent validation a detailed evaluation of noise variance is pivotal. In this respect, various studies have been reported to determine sources of variance and to provide tools for quantification $[14,25,31,34]$. The aim of this work is the development of a new tool for evaluation of spectral quality to improve future biomarker discovery studies. To this end, for further calculations the total signal variance $\sigma_{m}^{2}$ was estimated as follows. For one single MALDI target plate the noise levels in TOF- and FTICR profiles were determined for each of the five considered peptides as an average from 10 different samples (summarized in Table 1). Thus, $\sigma_{m}^{2}$ was considered constant at a specific $m / z$ value for each sample on one MALDI target plate. Based on visual inspection of the spectrum, an "empty" $\mathrm{m} / \mathrm{z}$ region was selected for each peptide used for alignment to calculate a corresponding $\sigma_{m}^{2}$ value. Using these $\sigma_{m}^{2}$ values the deviations between estimated and observed isotopic distributions were determined and summed to one single $Q C$ value for each spectrum according to eq 1 :

$$
(\mathrm{QC} \text {-value })^{2}=\sum_{p=1}^{p} \sum_{m=1}^{M} \frac{\left(I_{m}^{*}(\text { est. })-I_{m}^{*}(\text { obs. })\right)^{2}}{\sigma_{m}^{2}}
$$

in which $\mathrm{P}$ and $\mathrm{M}$ are the number of peptides and isotopes considered per peptide, respectively; $I_{m}^{*}(e s t$. and $I_{m}^{*}(o b s$.$) are the intensities of the estimated and$ observed isotopic peaks normalized for the sum of the intensities of the first three isotopic peaks in the corresponding isotopic distributions; the $\sigma_{m}^{2}$ value is the random variance noise calculated in a small set of analyzed spectra.

As an example, the QC values of 384 mass spectra (quadruplicate spots of 96 different serum samples on one MALDI target plate) are depicted in Figure 4. Note that a low QC value corresponds with a high quality spectrum. For most samples with a high quality profile, a similar QC value was observed for the four replicates, as exemplified for S21 in Figure 4. From this it was be concluded that MALDI-spotting followed by mass analysis showed good repeatability. QC based on isotopic distribution is a powerful tool for the evaluation of samples that yield low quality spectra. In some cases, only one out of four spectra was poor, as exemplified for S83, in other cases three out of four replicates were 


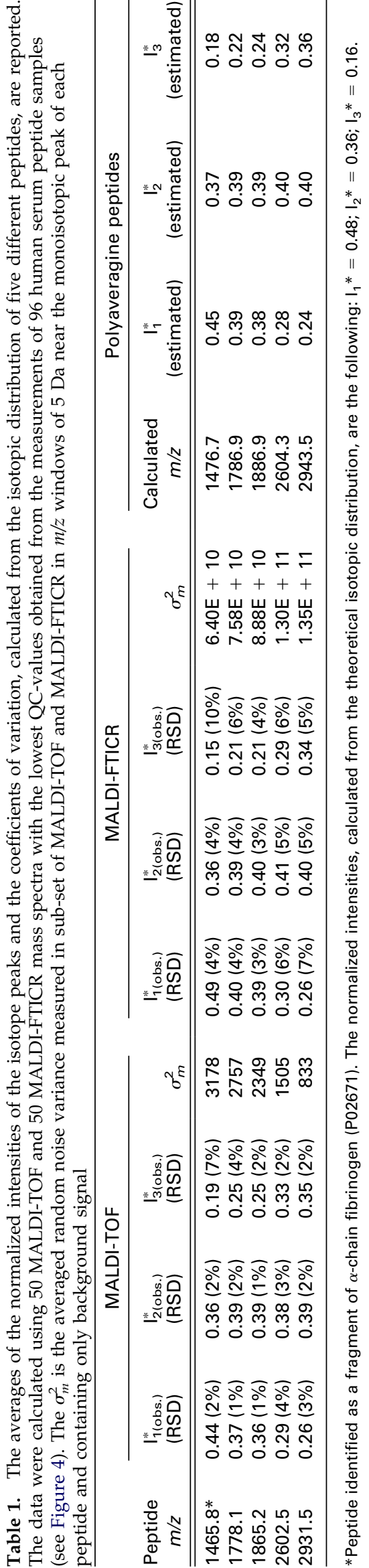

poor (e.g., S4 and S49). For this reason it is proposed to select the "best" spectrum of each sample for statistical analysis, i.e., with the lowest $\mathrm{QC}$ value. In cases that all four replicates yielded empty spectra (e.g., S58) or showed poor QC values (indicated with circled sample codes in Figure 4) the sample was excluded from further analysis. Applying the selection of one high quality spectrum for each sample ensures standardized further statistics, whereas upon considering all replicate spots the final profile is obtained after averaging of two, three or four spectra.

Another way to evaluate the similarity of multiple MALDI profiles is using the dot-product similarity to a reference spectrum as proposed by Yergey and coworkers [35]. In such a comparison, all signal is taken into account, including background peaks. The quality of spectra has been evaluated in detail counting the number of peaks in each replicate measurement [50]. Note that the number of peaks in a spectrum may be high as a result of contaminants and as such is earmarked "good quality". The same problem will arise upon using total ion intensities, which is exemplified in Figure 5. Here, the FTICR spectrum of no. 3 contains mostly polymers with high signal-to-noise ratios and will result in a false positive. As an alternative, peak picking algorithms are widely used to determine intensities of specific compounds in a spectrum. In combination with peak picking the quality of one single isotope distribution can be determined using the "sophisticated numerical annotation procedure" or SNAP (Bruker). When summarizing the SNAP quality factors of the five selected peptides, a similar trend as in our proposed quality values was observed. However, the extraction of SNAP quality factors from large numbers of mass spectra is time-consuming due to necessary peak picking and deconvolution of each spectrum. Moreover, in the case of a low quality spectrum these processes are often not robust and make automation more difficult. When manually applying SNAP on the same five peptides used for alignment, out of the four replicates in Figure 4, the same profiles were selected as low quality.

\section{Comparative Analysis of MALDI-TOF and MALDI-FTICR Spectra}

For the purpose of comparing TOF and FTICR serum peptide profiles, the same 192 spots on a MALDI target plate were measured both on a MALDI-TOF and a MALDI-FTICR instrument. The QC value for each TOF spectrum as well as for each FTICR spectrum was determined according to eq 1 . The results are plotted in Figure 5 after sorting. Here, it can be observed that a small fraction of the spectra showed a drastically increased QC value that is indicative for a poor spectral quality. It was verified that all TOF-spectra of MALDIspots with high QC value also yielded low quality FTICR-spectra. In other words, trend analysis of both 

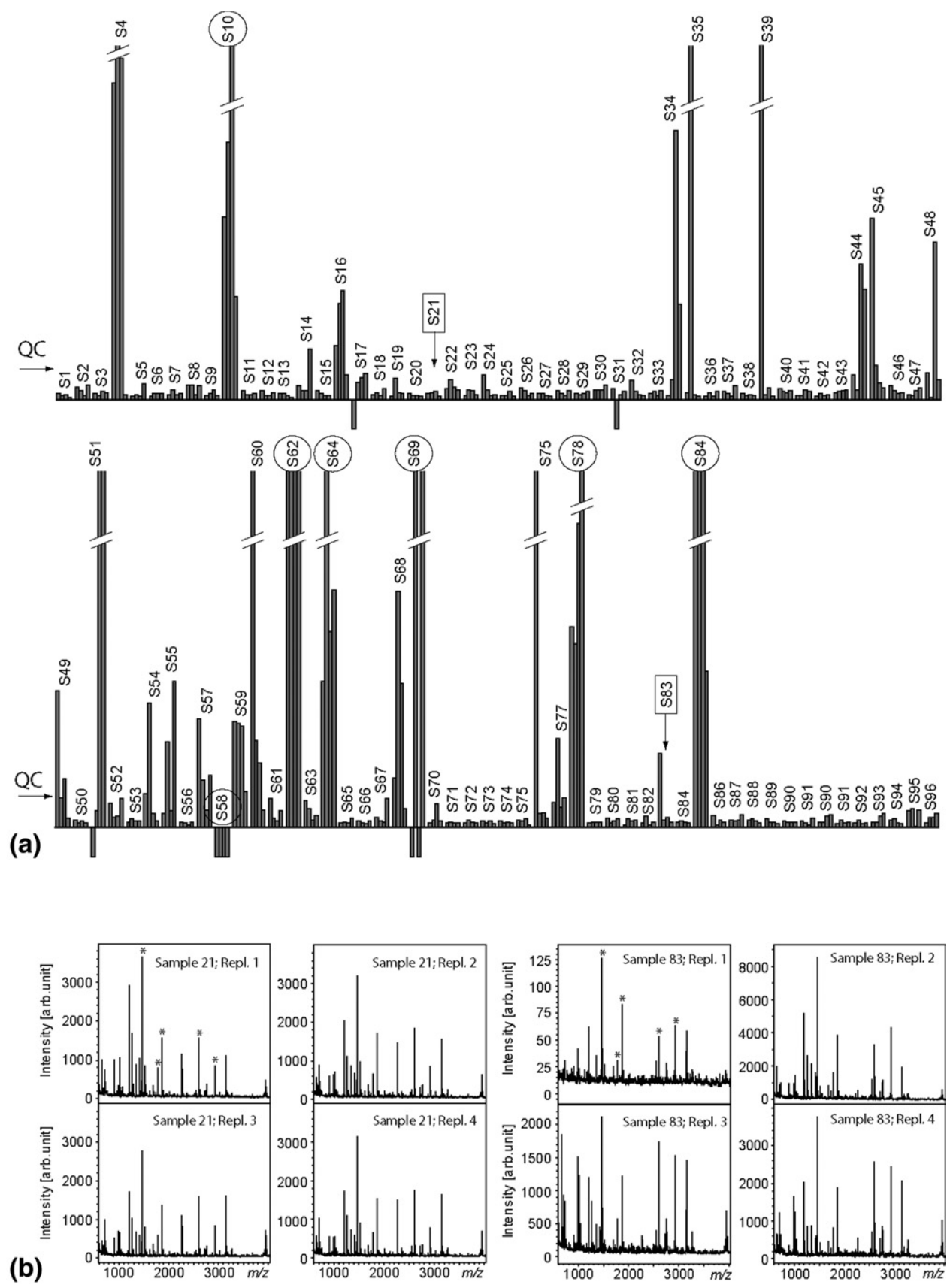

Figure 4. (a) Histogram of 384 QC values of 96 human serum samples measured in quadruplicate with MALDI-TOF. The low values (short bars) indicate high quality spectra while a negative value is automatically assigned when an empty spectrum is recorded. Based on these QC values it was found that from the total batch of recorded peptide profiles, 63 spectra were of poor quality, i.e., $16 \%$ of the data were not suitable for further analysis. The cut-off point is indicated with an arrow in the plot. For seven samples, all four replicates yielded a poor quality spectrum (indicated with a circle over the sample code). As a result, when taking into account only the spectrum with the highest quality for each sample, $93 \%$ of the data were of high quality and included for further analysis. (b) Two examples of four replicate MALDI-TOF spectra of samples S21 and S83. The calculation of the QC values for each of these peptide profiles allowed the selection of one spectrum for further analysis. Ordering according to the QC method resulted in sample 23 in 4-1-2-3 and in sample 83 in 4-2-3-1. Ordering according to the sum of SNAP quality factors of the same five peptides (indicated with an asterisk in the first spectrum) resulted for samples 23 and 83 in 1-2-4-3 and 2-4-3-1, respectively.

TOF- and FTICR data resulted in a similar selection of low quality data. It is important to note that the choice of a cut-off point in Figure 5 will be different for each
MALDI target plate considered. Any chosen cut-off point for the discrimination between the high and the poor quality spectra based on QC values will introduce 

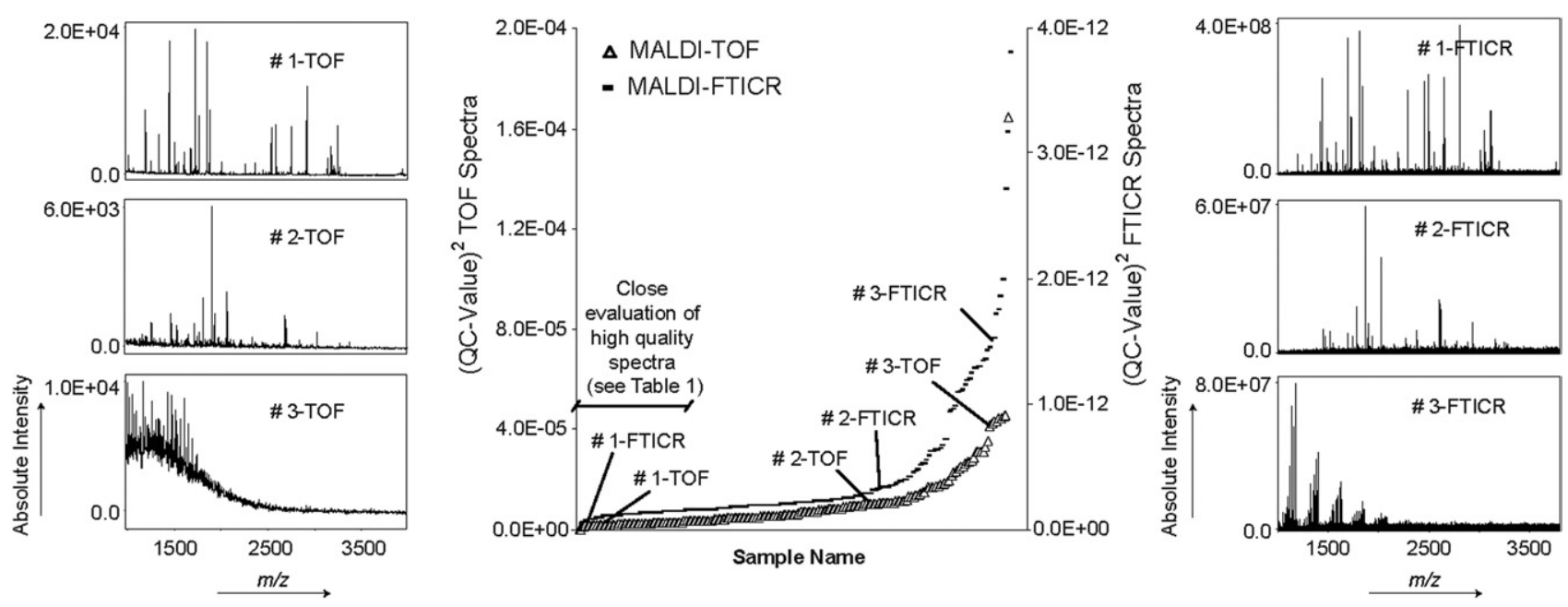

Figure 5. Plot of QC values derived from 192 MALDI-TOF and MALDI-FTICR human serum peptide profiles. These 192 profiles were obtained from 96 different samples after duplicate spotting on a MALDI target plate. The QC values are calculated applying eq 1 to the isotopic distributions of five peptides selected in Figure 2. After sorting the MALDI spot numbers by their QC values, clear trends in peptide profile quality are observed.

false-positive and false-negative results. For a quantitative analysis, the normalized intensities estimated from the polyaveragine peptides, the average ratios of the isotopic distributions and their coefficients of variation were evaluated for the five peptides discussed earlier (see Figure 2) and the results are summarized in Table 1. In this evaluation, the 50 MALDI-TOF and the 50 MALDI-FTICR mass spectra with lowest QC values were selected from the results obtained from 192 peptide profiles in Figure 5. It can be seen from Table 1 that the observed isotope ratios were in good agreement with those estimated from the polyaveragine distribution. For example, the averaged isotope ratio of the first and the second peak in the distribution of the peptide at $\mathrm{m} / \mathrm{z} 1865$ was 1.1 in the TOF spectra and 1.0 in the FTICR, which is in good agreement with the estimated value of 1.0 from the polyaveragine peptide with $\mathrm{m} / \mathrm{z}$ 1886. The results in Table 1 show that the coefficients of variation of the first isotope ratios were within $6 \%$ and $13 \%$ for MALDI-TOF and MALDI-FTICR, respectively.

With respect to the possibility of overlap of multiple peptides, it was observed from MALDI-FTICR data that the $\mathrm{m} / \mathrm{z}$ regions of the five peptides manually selected for the QC method contained a singly species. Finally, if in the serum, glycopeptides would be present in which the contribution of the glycan part is relatively large, these would be lost upon the RPC18 extraction. This leaves inherent deviations from the polyaveragine peptide as the only explanation for different isotopic distributions in the five peptides. Thus, the observed deviations can be attributed to the differences between the real and the estimated composition.

\section{Conclusions}

In this study, the isotopic distributions of a small set of peptides were used as a quality control parameter for the evaluation of MALDI-TOF and MALDI-FTICR spectra. This novel QC method allows a semiautomated selection of a good spectrum from replicate measurements of one sample and for the removal of low quality spectra from further statistical analysis, which is necessary for large-scale study. The isotopic distributions of five peptides (present in all serum samples) were determined and evaluated in both high-resolution MALDI-TOF and ultrahigh resolution MALDI-FTICR spectra. The selected isotope patterns were compared with polyaveragine distributions to calculate a quality control value for each single mass spectrum. Sorting the obtained QC values allowed the selection of the best MALDI spectrum from replicate spots and the removal of low quality spectra from further data analysis. Examples of odd peptide isotope distributions in MALDI-TOF spectra could be rationalized from corresponding ultrahigh resolution FTICR profiles that showed overlap of different peptides. As was earlier shown and has been discussed by other groups, multiple factors can invalidate the results of peptide profiling studies. To obtain a confident result, the full workflow of a profiling study should be highly standardized. The novel QC parameter used in this study is objective and facilitates further data analysis in large scale clinical studies. The proposed QC value can be determined for any isotopically-resolved MALDI-profile, i.e., this method is independent of the type of instrument, the manufacturer, or the software used for data processing. The use of this parameter in combination with other quality criteria that take into account signal-to-noise, reproducibility, and baseline threshold should result in a better selection of high-quality spectra. The integration of all such metrics for quality evaluation into one single method is under development. 


\section{Acknowledgments}

The authors acknowledge that this work is part of the research program "Decrease Colorectal Cancer Death," which is financially supported by the Center for Translational Molecular Medicine (CTMM).

\section{References}

1. Anderson, N. L.; Anderson, N. G. The Human Plasma Proteome. Mol. Cell. Proteom. 2002, 1, 845-867.

2. Aebersold, R.; Mann, M. Mass Spectrometry-Based Proteomics. Nature 2003, 422, 198-207.

3. Syka, J. E. P.; Coon, J. J.; Schroeder, M. J.; Shabanowitz, J.; Hunt, D. F. Peptide and Protein Sequence Analysis by Electron Transfer Dissociation Mass Spectrometry. Proc. Natl. Acad. Sci. U.S.A. 2004, 101(26), 9528-9533.

4. Coon, J. J. Collisions or Electrons? Protein Sequence Analysis in the 21st Century. Anal. Chem. 2009, 81(9), 3208-3215.

5. Makarov, A.; Denisov, E.; Kholomeev, A.; Balschun, W.; Lange, O.; Strupat, K.; Horning, S. Performance Evaluation of a Hybrid Linear Ion Trap/Orbitrap Mass Spectrometer. Anal. Chem. 2006, 78, 2113-2120.

6. Makarov, A.; Denisov, E.; Lange, O. Performance Evaluation of a High-field Orbitrap Mass Analyzer Source. J. Am. Soc. Mass Spectrom. 2009, 20, 1391-1396

7. Schäfer, R. UltrafleXtreme: Redefining MALDI-TOF-TOF Mass Spectrometry Performance. LC GC Europe 2009, (Suppl. S), 26-27.

8. Palmblad, M.; Drijfhout, J. W.; Deelder, A. M. High Resolution Mass Spectrometry for Rapid Characterization of Combinatorial Peptide Libraries. J. Comb. Chem. 2010, 12, 65-68.

9. Frank, R.; Hargreaves, R. Clinical Biomarkers in Drug Discovery and Development. Nat. Rev. Drug Discovery. 2003, 2, 566-580.

10. Palmblad, M.; Tiss, A.; Cramer, R. Mass Spectrometry in Clinical Proteomics-from the Present to the Future. Proteomics Clin. Appl. 2009, 3, 6-17.

11. Villanueva, J.; Philip, J.; Entenberg, D.; Chaparro, C. A.; Tanwar, M. K.; Holland, E. C.; Tempst, P. Serum Peptide Profiling by Magnetic Particle-Assisted, Automated Sample Processing and MALDI-TOF Mass Spectrometry. Anal. Chem. 2004, 76, 1560-1570.

12. Veenstra, T. D. Global and Targeted Quantitative Proteomics for Biomarker Discovery. J. Chromatogr. B 2007, 847, 3-11.

13. Petricoin, E. F.; Ardekani, A. M.; Hitt, B. A.; Levine, P. J.; Fusaro, V. A.; Steinberg, S. M.; Mills, G. B.; Simone, C.; Fishman, D. A.; Kohn, E. C.; Liotta, L. A. Use of Proteomic Patterns in Serum to Identify Ovarian Cancer Lancet 2002, 359, 572-577.

14. Callesen, A. K.; Vach, W.; Jørgensen, P. E.; Cold, S.; Mogensen, O.; Kruse, T. A.; Jensen, O. N.; Madsen, J. S. Reproducibility of Mass Spectrometry Based Protein Profiles for Diagnosis of Breast Cancer Across Clinical Studies: A Systematic Review. J. Proteome Res. 2008, 7, 1395-1402.

15. Barnea, E.; Sorkin, R.; Ziv, T.; Beer, I.; Admon, A. Evaluation of Prefractionation Methods as a Preparatory Step for Multidimensional Based Chromatography of Serum Proteins. Proteomics 2005, 5, 33673375.

16. Luque-Garcia, J. L.; Neubert, T. A. Sample Preparation for Serum/ Plasma Profiling and Biomarker Identification by Mass Spectrometry. J.Chromatogr. A 2007, 1153, 259-276.

17. Faca, V.; Pitteri, S. J.; Newcomb, L.; Glukhova, V.; Phanstiel, D.; Krasnoselsky, A.; Zhang, Q.; Struthers, J.; Wang, H.; Eng, J.; Fitzgibbon, M.; McIntosh, M.; Hanash, S. Contribution of Protein Fractionation to Depth of Analysis of the Serum and Plasma Proteomes. J. Proteome Res. 2007, 6, 3558-3565.

18. Nissum, M.; Foucher, A. L. Analysis of Human Plasma Proteins: A Focus on Sample Collection and Separation Using Free-Flow Electrophoresis. Expert Rev. Proteom. 2008, 5(4), 571-587.

19. Mauri, P.; Petretto, A.; Cuccabita, D.; Basilico, F.; Di Silvestre, D.; Levreri, I.; Melioli, G.; Fractionation Techniques Improve the Proteomic Analysis of Human Serum. Current Pharmaceut. Anal. 2008, 4, 69-77.

20. Baumann, S.; Ceglarek, U.; Fiedler, G. M.; Lembcke, J.; Leichtle, A.; Thiery, J. Standardized Approach to Proteome Profiling of Human Serum Based on Magnetic Bead Separation and Matrix-Assisted Laser Desorption/Ionization Time-of-Flight Mass Spectrometry. Clin. Chem. 2005, 51(6), 973-980.

21. Jimenez, C. R.; Koel-Simmelink, M.; Pham, T. V.; van der Voort, L.; Teunissen, C. E. Endogenous Peptide Profiling of Cerebrospinal Fluid by MALDI-TOF Mass Spectrometry: Optimization of Magnetic BeadBased Peptide Capture and Analysis of Preanalytical Variables. Proteom. Clin. Appl. 2007, 1, 1385-1392.

22. Alagaratnam, S.; Mertens, B. J. A.; Dalebout, J. C.; Deelder, A. M.; van Ommen, G. B.; den Dunnen, J. T.; 't Hoen, P. A. C. Serum Protein Profiling in Mice: Identification of Factor XIIIa as a Potential Biomarker for Muscular Dystrophy. Proteomics 2008, 8, 1552-1563.

23. Rai, A. J.; Vitzthum, F. Effects of Preanalytical Variables on Peptide and Protein Measurements in Human Serum and Plasma: Implications for Clinical Proteomics. Expert Rev. Proteom. 2006, 3(4), 409-426.

24. Penno, M. A. S.; Ernst, M.; Hoffmann, P. Optimal Preparation Methods for Automated Matrix Assisted Laser Desorption/Ionization Time-of-
Flight Mass Spectrometry Profiling of Low Molecular Weight Proteins and Peptides. Rapid Commun. Mass Spectrom. 2009, 23, 2656-2662.

25. Callesen, A. K.; Madsen, J. S.; Vach, W.; Kruse, T. A.; Mogensen, O.; Jensen, O. N. Serum Protein Profiling by Solid Phase Extraction and Mass Spectrometry: A Future Diagnostics Tool? Proteomics 2009, 9, 1428-1441.

26. Villanueva, J.; Lawlor, K.; Toledo-Crow, R.; Tempst, P. Automated Serum Peptide Profiling. Nature Protoc. 2006, 1(2), 880-891.

27. Jimenez, C. R.; El Filali, Z.; Knol, J. C.; Hoekman, K.; Kruyt, F. A. E.; Giaccone, G.; Smit, A. B.; Li, K. W. Automated Serum Peptide Profiling Using Novel Magnetic C18 Beads Off-Line Coupled to MALDI-TOFMS. Proteomics Clin. Appl. 2007, 1, 598-604.

28. Villanueva, J.; Nazarian, A.; Lawlor, K.; Tempest, P. Monitoring Peptidase Activities in Complex Proteomes by MALDI-TOF Mass Spectrometry. Nature Protoc. 2009, 4(8), 1167-1183.

29. Dekker, L. J.; Boogerd, W.; Stockhammer, G.; Dalebout, J. C.; Siccama, I.; Zheng, P.; Bonfrer, J. M.; Verschuuren, J. J.; Jenster, G.; Verbeek, M. M. Luider, T. M.; Sillevis Smitt, P. A. MALDI-TOF Mass Spectrometry Analysis of Cerebrospinal Fluid Tryptic Peptide Profiles to Diagnose Leptomeningeal Metastases in Patients with Breast Cancer. Mol. Cell. Proteom. 2005, 4, 1341-1349.

30. de Noo, M. E.; Mertens, B. J. A.; Özalp, A.; Bladergroen, M. R.; van der Werff, M. P. J.; van de Velde, C. J. H.; Deelder, A. D.; Tollenaar, R. A. E. M. Detection of Colorectal Cancer Using MALDI-TOF Serum Protein Profiling. Eur. J. Cancer 2006, 42, 1068-1076.

31. de Noo, M. E.; Tollenaar, R. A. E. M.; Özalp, A.; Kuppen, P. J. K. Bladergroen, M. R.; Eilers, M. R. P.; Deelder, A. M. Reliability of Human Serum Protein Profiles Generated with C8 Magnetic Beads Assisted MALDI-TOF Mass Spectrometry. Anal. Chem. 2005, 77, 7232-7241.

32. Stoop, M. P.; Dekker, L. J.; Titulaer, M. K.; Lamers, R. A. N.; Burgers, P. C.; Sillevis Smitt, P. A. E.; van Gool, A. J.; Luider, T. M.; Hintzen, R. Q. Quantitative Matrix-Assisted Laser Desorption Ionization-Fourier Transform Ion Cyclotron Resonance (MALDI-FT-ICR) Peptide Profiling and Identification of Multiple-Sclerosis-Related Proteins. J. Proteome Res. 2009, 8, 1404-1414.

33. Römpp, A.; Dekker, L.; Taban, I.; Jenster, G.; Boogerd, W.; Bonfrer, H. Spengler, B.; Heeren, R.; Sillevis Smitt, P. A.; Luider, T. M. Identification of Leptomeningeal Metastasis-Related Proteins in Cerebrospinal Fluid of Patients with Breast Cancer by a Combination of MALDI-TOF MALDI-FTICR and nanoLC-FTICR MS. Proteomics 2007, 7, 474-481.

34. Harrington, P. B.; Vieira, N. E.; Chen, P.; Espinoza, J.; Nien, J. K Romero, R.; Yergey, A. L. Proteomic Analysis of Amniotic Fluids Using Analysis of Variance-Principal Component Analysis and Fuzzy RuleBuilding Expert Systems Applied to Matrix-Assisted Laser Desorption/ Ionization Mass Spectrometry. Chemomet. Intell. Lab. Syst. 2006, 82, 283-293.

35. Olson, M. T.; Blank, P. S.; Sackett, D. L.; Yergey, A. L. Evaluating Reproducibility and Similarity of Mass and Intensity Data in Complex Spectra-Applications to Tubulin. J. Am. Soc. Mass Spectrom. 2008, 19, 367-374.

36. Cairns, D. A.; Perkins, D. N.; Stanley, A. J.; Thompson, D.; Barrett, J. H. Selby, P. J.; Banks, R. E. Integrated Multi-Level Quality Control for Proteomic Profiling Studies Using Mass Spectrometry. BMC Bioinf. 2008, 9, 519.

37. Bons, J. A. P.; de Boer, D; van Dieijen-Visser, M. P; Wodzig, W. K. W. H. Standardization of Calibration and Quality Control Using Surface Enhanced Laser Desorption Ionization-Time of Flight-Mass Spectrometry. Clin. Chim. Acta. 2006, 366, 249-256.

38. McLerran, D.; Grizzle, W. E.; Feng, Z.; Bigbee, W. L.; Banez, L. L.; Cazares, L. H.; Chan, D. W.; Diaz, J.; Izbicka, E.; Kagan, J.; Malehorn, D. E.; Malik, G.; Oelschlager, D.; Partin, A.; Randolph, T.; Rosenzweig, N.; Srivastava, S.; Srivastava, S.; Thompson, I. M.; Thornquist, M.; Troyer, D.; Yasui, Y.; Zhang, Z.; Zhu, L.; Semmes, O. J. Analytical Validation of Serum Proteomic Profiling for Diagnosis of Prostate Cancer: Sources of Sample Bias. Clin. Chem. 2008, 54(1), 44-52.

39. Albrethsen, J. Reproducibility in Protein Profiling by MALDI-TOF Mass Spectrometry. Clin. Chem. 2007, 53(5), 852-858.

40. Callesen, A. K.; DePont Christensen, R.; Madsen, J. S.; Vach, W.; Zapico E.; Cold, S.; Jørgensen, P. E.; Mogensen, O.; Kruse, T. A.; Jensen. O. N. Reproducibility of Serum Protein Profiling by Systematic Assessment Using Solid-Phase Extraction and Matrix-Assisted Laser Desorption/ Ionization Mass Spectrometry. Rapid Commun. Mass Spectrom. 2008, 22, 291-300.

41. Senko, M. W.; Beu, S. C.; McLafferty, F. W. Determination of Monoisotopic Masses and Ion Populations for Large Biomolecules from Resolved Isotopic Distributions. J. Am. Soc. Mass Spectrom. 1995, 6, 229-233.

42. McDonnell, L.A.; van Remoortere, A.; van Zeijl, R.; Dalebout, H.; Bladergroen, M.R.; Deelder, A.M. Automated Imaging MS: Toward High Throughput Imaging Mass Spectrometry. J. Proteom. 2010, 73, 1279-1282.

43. Selman, M. H. J.; McDonnell, L. A.; Palmblad, M.; Ruhaak, L. R. Deelder A. M.; Wuhrer, M. Immunoglobulin G Glycopeptide Profiling by Matrix-Assisted Laser Desorption Ionization Fourier Transform Ion Cyclotron Resonance Mass Spectrometry. Anal. Chem. 2010, 82, 1073 1081.

44. Rockwood, A. L.; Haimi, P. Efficient Calculation of Accurate Masses of Isotopic Peaks. J. Am. Soc. Mass Spectrom. 2006, 17, 415-419.

45. Villanueva, J.; Shaffer, D. R.; Philip, J.; Chaparro, C. A.; ErdjumentBromage, H.; Olshen, A. B.; Fleisher, M.; Lilja, H.; Brogi, E.; Boyd, J.; Sanchez-Carbayo, M.; Holland, E. C.; Cordon-Cardo, C.; Scher, H. I.; 
Tempst, P. Differential Exoprotease Activities Confer Tumor-Specific Serum Peptidome Patterns. J. Clin. Investig. 2006, 116(1), 271-284.

46. Breen, E. J.; Hopwood, F. G.; Williams, K. L.; Wilkins, M. R. Automatic Poisson Peak Harvesting for High-Throughput Protein Identification. Electrophoresis 2000, 21, 2243-2251.

47. Valkenborg, D.; Jansen, I.; Burzykowski, T. A Model-Based Method for the Prediction of the Isotopic Distribution of Peptides. I. Am. Soc. Mass Spectrom. 2008, 19, 703-712.

48. Sachon, E.; Matheron, L.; Clodic, G.; Blascoa, T.; Bolbacha, G. MALDI TOF-TOF Characterization of a Light Stabilizer Polymer Contaminant from Polypropylene or Polyethylene Plastic Test Tubes. J. Mass Spectrom. 2010, 45, 43-50.

49. Palmblad, M.; Buijs, J.; Håkansson, P. Automatic Analysis of Hydrogen/Deuterium Exchange Mass Spectra of Peptides and Proteins Using Calculations of Isotopic Distributions. J. Am. Soc. Mass Spectrom. 2001, 12, 1153-1162.

50. Dekker, L. J. Dalebout J C. Siccama, I. Jenster G. Sillevis Smitt, P. A. Luider, T. M. A New Method to Analyze Matrix-Assisted Laser Desorption/Ionization Time-of-Flight Peptide Profiling Mass Spectra. Rapid Commun. Mass Spectrom. 2005, 19, 865-870. 